\title{
A Primer to proliferation
}

Principles of Cell Proliferation

by John K. Keath

Blackwell Science $\cdot$ December 2000

Paperback $£ 22.50 / \$ 37.95$

Michael Brandeis

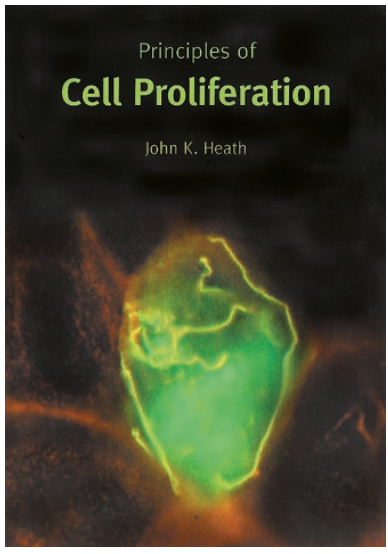

B ary division of cells is one of the most basic features of life but also one of its biggest miracles. For a cell to divide it has to replicate its genome and to equally distribute it to both its daughter cells. In all eukaryotes, genome replication (S-phase) and division (mitosis) take place in a sequential and non-overlapping manner. Moreover, in most cells replication and mitosis are separated by the so called 'gap phases' G1 and G2. During the G1 phase, which precedes replication, the cell has to reach the all-important decision whether to commit itself to another division cycle. Normal cells are responsible enough not to reach such a vital decision, which could affect the entire organism, on their own. They require the input of external signals, in the form of molecules secreted by other cells, to decide whether to replicate and divide, or to remain quiescent for the time being. Once a decision has been made to enter another round of replication and division, external signals are no longer required. The best way to define cancer cells is to say that they act irresponsibly and do not care about external signals when they decide to proliferate. This characteristic of cancer cells is what has made cell proliferation into such an important and intensively studied discipline. The closely related, but not identical, field of cell-cycle research is more concerned with the processes the cell undergoes after it reached a positive decision to cycle.

The insight into how cells proliferate and cycle has made revolutionary progress over recent years. How little of what we know today was known just ten years ago, and how much will our entire view be changed in just another ten years? It must therefore be a hard and ungrateful job to write a book describing such a fast-moving field. State of the art textbooks dedicated to cell proliferation are indeed a rarity and anybody who attempts to write one is doing a great service.

Principles of Cell Proliferation is written in a simple but in no way simplistic man- ner. It is one of the great achievements of the author that he managed to explain very complex issues of cell proliferation in a way that requires very little prior knowledge in biology. The book is suitable for graduate, and even undergraduate students, and is short enough to be read from cover to cover. It is also very useful for anybody working in related or distant fields to get acquainted with the principles of cell proliferation.

The book starts with a short introduction to major concepts such as the phases of the cell cycle, quiescence, senescence, stem cells and so on. It then takes a systematic approach starting from the external signal and following it to the final effect. A chapter describing the many different growth factors is followed by one detailing the large number of transmembrane receptors and the way they relay the signal into the cell. These signals are next transmitted and integrated in a very complex and highly interwoven network of kinases.

The next chapter shows how these kinases eventually activate transcription factors leading to the expression of proteins that are required for entry in the cell cycle. The sixth chapter gives a very schematic description of the cell-cycle engine and is somewhat dated. Unfortunately it does not mention many of the most exciting insights gained in recent years into how genome replication is regulated, or how protein degradation regulates cell-cycle events. Oncogenes and anti-oncogenes described in the next two chapters describe proteins that are essential for normal regulation of cell proliferation. If these proteins go wrong in any way, cells end up proliferating wildly and causing cancer. Once cells enter the cell cycle there is no way back. The continued health of the organism thus requires that if anything goes wrong and cannot be repaired the cell must be eliminated. This elimination takes place by a suicidal pathway called programmed cell death that is described in the final chapter.

The many illustrations in the book can be divided into two types. The first consists of simple but excellent line drawings that help very much to understand the pathways described in the text. The second type consists of grey structural drawings of many of the described proteins. Without proper legends they often serve only as decorations and will contribute little to most readers.

It is easy to criticize any such book for omitting many important issues and, as correctly pointed out in the introduction, the choice of subjects is very much a personal matter. However, this is on the whole a legible and very useful book to teachers and students alike.

Michael Brandeis is in the Department of Genetics, The Institute of Life Sciences, The Hebrew University of Jerusalem, Jerusalem 91904, Israel

e-mail:brandeis@leonardo.huji.ac.il

\section{New in Hardback}

Stem Cell Biology

edited by D. R. Marshak, R. L. Gardner and D. Gottleib

Cold Spring Harbor Laboratory Press, $\mathfrak{E} 85 / \$ 115$

The Story of Taxol

by Jordan Goodman and Vivien Walsh Cambridge University Press, £18.95/\$27.95

Cause and Correlation in Biology: A

User's Guide to Path Analysis,

Structural Equations and Casual

Inference

by John William Shipley

Cambridge University Press, $£ 45 / \$ 69.95$

\section{New in Paperback}

The Recombinant DNA Controversy: A Memoir

by D. Fredrickson

ASM, $£ 32.50 / \$ 39.95$

Measuring Calcium and Calmodulin Inside and Outside Cells edited by Ole H. Petersen Springer, $£ 44.50 / \$ 69.95$ 